\title{
BMJ Open Retrospective cohort study of admission timing and mortality following COVID-19 infection in England
}

\author{
Ahmed Alaa, ${ }^{1}$ Zhaozhi Qian, ${ }^{2}$ Jem Rashbass, ${ }^{3}$ Jonathan Benger (D) , ${ }^{3}$ \\ Mihaela van der Schaar ${ }^{2}$
}

To cite: Alaa A, Qian Z, Rashbass J, et al. Retrospective cohort study of admission timing and mortality following COVID-19 infection in England. BMJ Open 2020;10:e042712. doi:10.1136/ bmjopen-2020-042712

- Prepublication history for this paper is available online. To view these files, please visit the journal online (http://dx.doi org/10.1136/bmjopen-2020042712).

Received 14 July 2020 Revised 27 October 2020 Accepted 10 November 2020

Check for updates

(c) Author(s) (or their employer(s)) 2020. Re-use permitted under CC BY-NC. No commercial re-use. See rights and permissions. Published by BMJ.

${ }^{1}$ University of California, Los Angeles, California, USA

${ }^{2}$ Centre for Mathematical Sciences, Cambridge University, Cambridge, UK

${ }^{3} \mathrm{NHS}$ Digital, Leeds, UK

Correspondence to

Professor Jonathan Benger;

JBenger@nhs.net

\section{ABSTRACT}

Objectives We investigated whether the timing of hospital admission is associated with the risk of mortality for patients with COVID-19 in England, and the factors associated with a longer interval between symptom onset and hospital admission.

Design Retrospective observational cohort study of data collected by the COVID-19 Hospitalisation in England Surveillance System (CHESS). Data were analysed using multivariate regression analysis.

Setting Acute hospital trusts in England that submit data to CHESS routinely.

Participants of 14150 patients included in CHESS until 13 May 2020, 401 lacked a confirmed diagnosis of COVID-19 and 7666 lacked a recorded date of symptom onset. This left 6083 individuals, of whom 15 were excluded because the time between symptom onset and hospital admission exceeded 3 months. The study cohort therefore comprised 6068 unique individuals.

Main outcome measures All-cause mortality during the study period.

Results Timing of hospital admission was an independent predictor of mortality following adjustment for age, sex, comorbidities, ethnicity and obesity. Each additional day between symptom onset and hospital admission was associated with a $1 \%$ increase in mortality risk (HR 1.01; $p<0.005)$. Healthcare workers were most likely to have an increased interval between symptom onset and hospital admission, as were people from Black, Asian and minority ethnic (BAME) backgrounds, and patients with obesity.

Conclusion The timing of hospital admission is associated with mortality in patients with COVID-19. Healthcare workers and individuals from a BAME background are at greater risk of later admission, which may contribute to reports of poorer outcomes in these groups. Strategies to identify and admit patients with highrisk and those showing signs of deterioration in a timely way may reduce the consequent mortality from COVID-19, and should be explored.

\section{BACKGROUND}

On 31 December 2019, the WHO China country office was informed of 44 cases of pneumonia of unknown cause in Wuhan city, China. ${ }^{1}$ By 14 June 2020, more than 7.8 million cases of COVID-19 disease caused by the SARS-CoV-2 had been reported across

\section{Strengths and limitations of this study}

- We used all available data from a large observational data set, and analysed this using two multivariate regression models. Our findings are internally consistent and statistically and clinically significant.

- This research has several limitations. Of 13749 patients with a confirmed diagnosis of COVID-19, only 6083 could be included in the study, and the date of symptom onset was self-reported, rather than objectively verified.

- Our data are observational and should be interpreted with caution; the results may have been influenced by unmeasured confounders.

188 countries and regions, resulting in 431000 deaths. $^{2}$

Throughout the early stages of the pandemic clinicians treating patients with COVID-19 reported that infected individuals were tending to present later than expected to hospital services, with many profoundly hypoxic and requiring respiratory support soon after admission. This has been attributed to the phenomenon of 'silent hypoxia'; in COVID-19 infection, oxygen levels may fall dangerously low without an individual becoming aware of this. ${ }^{3}$ Another reason for later presentation in the UK may have been the public's desire to 'protect the National Health Service (NHS) ${ }^{4}{ }^{4}$ and also a tendency to stay away from hospitals because of concerns that there was a risk of coming into contact with the disease there. ${ }^{5}$ However, late presentation to hospital services delays the correction of hypoxia and the initiation of potentially life-saving treatments including respiratory support and dexamethasone. ${ }^{6}$

We hypothesised that later hospital admission may be associated with increased mortality. We also hypothesised that some patient groups may be more prone to later hospital admission, providing an opportunity to deliver interventions to those most at risk. 


\section{Objectives}

- To determine whether the timing of hospital admission is associated with mortality for patients diagnosed with COVID-19 infection.

- To identify the characteristics of patients with COVID-19 most at risk of later hospital admission.

\section{METHODS}

\section{Study design}

We completed a retrospective observational cohort study using data from the COVID-19 Hospitalisation in England Surveillance System (CHESS), established by Public Health England to collect routine data during the COVID-19 pandemic.

\section{Setting}

We included data collected by CHESS between 8 February and 13 April 2020. During this time, data returns were received from 94 of $152(62 \%)$ of all acute hospital trusts in England.

\section{Participants}

Participants were individuals included in the CHESS database during the study period. We excluded patients without a confirmed diagnosis of COVID-19, those for whom the date of symptom onset was not recorded and those in whom the recorded date of symptom onset was more than 3 months before or after the date of hospital admission (on the assumption that a data entry error had occurred).

\section{Variables}

A set of 23 case-level variables were identified for each patient, as follows.

\section{Demographic}

Age; sex; obesity; ethnicity (White, Asian, Black, other) and healthcare worker.

\section{Comorbidities}

Respiratory; asthma; diabetes; cardiovascular; hypertension; renal; liver; immunosuppressive disease and immunosuppressive treatment.

\section{Timing of hospitalisation}

The timing of hospitalisation was a continuous variable defined as the number of days between symptom onset and admission to hospital. Patients hospitalised before symptom onset were assumed to have been admitted for a reason other than COVID-19: either they were infected but asymptomatic on admission or they became infected while in hospital.

The primary outcome was all-cause mortality during the study follow-up period. Patients who remained in hospital at the end of the follow-up period (13 May 2020) were considered to be censored.

\section{Sample size}

This was a consecutive sample, using all available data.

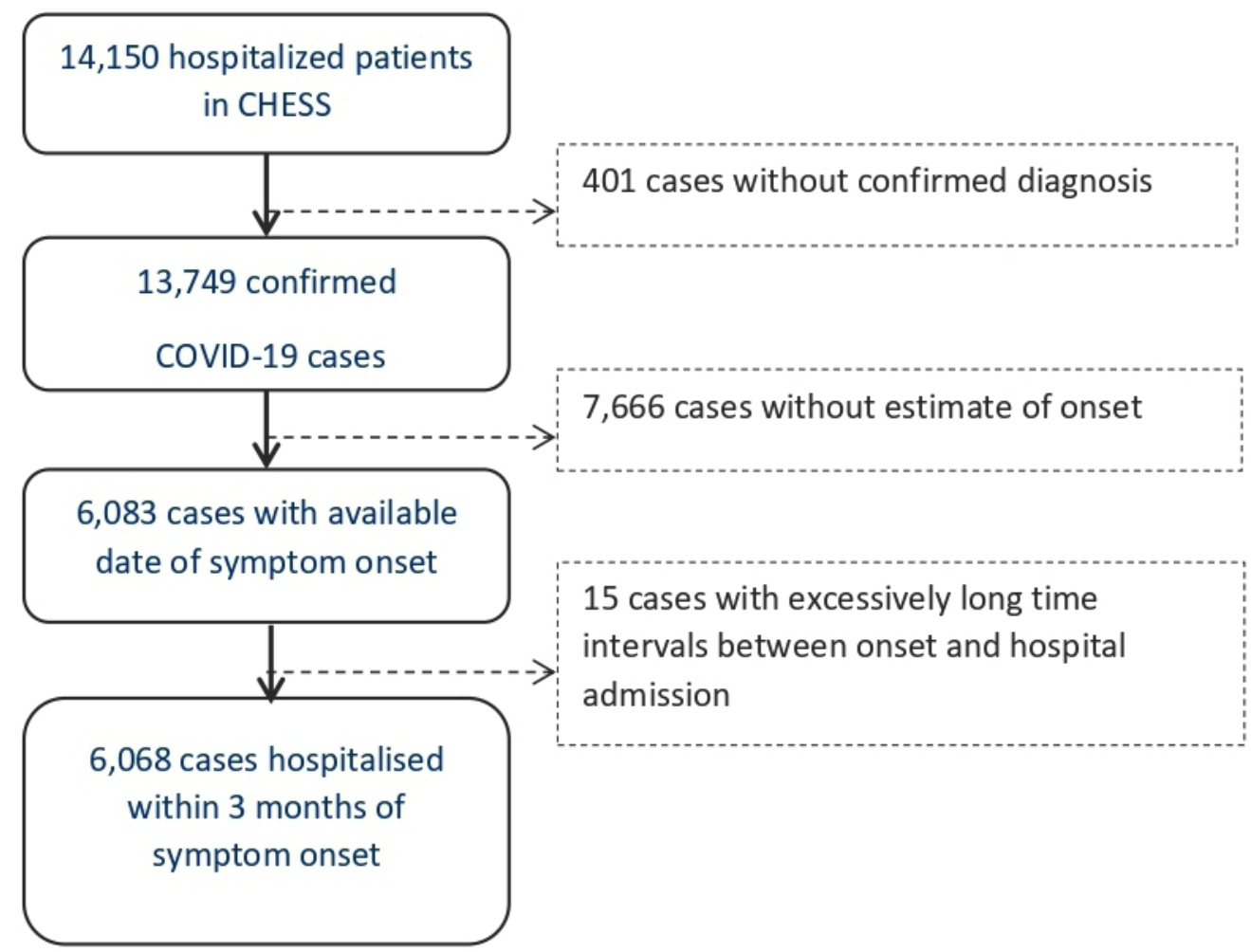

Figure 1 Patients' flow diagram. CHESS, COVID-19 Hospitalisation in England Surveillance System. 
Table 1 Comparison of the included cohort with all those recorded in the CHESS database

\begin{tabular}{lll}
\hline & $\begin{array}{l}\text { Analysis cohort } \\
(\mathbf{n = 6 0 6 8 )}\end{array}$ & $\begin{array}{l}\text { All CHESS } \\
\text { patients } \\
(\mathbf{n}=14150)\end{array}$ \\
\hline Median age & 66 years & 65 years \\
\hline Male sex & $61 \%$ & $60 \%$ \\
\hline Obesity & $12 \%$ & $7 \%$ \\
\hline White ethnicity & $58 \%$ & $46 \%$ \\
\hline Asian ethnicity & $6.6 \%$ & $5.1 \%$ \\
\hline Black ethnicity & $19 \%$ & $15 \%$ \\
\hline Number of comorbidities & 1.0 & 0.65 \\
\hline
\end{tabular}

CHESS, COVID-19 Hospitalisation in England Surveillance System.

\section{Statistical methods}

Two multivariate regression models were used to estimate the impact of admission timing on patient mortality risk, and identify the patient characteristics associated with later hospital admission. A Cox proportional hazards regression model using the 22 case-level variables was fit with the patients' survival and follow-up time as outcomes. In addition, a binary logistic regression model was fit to infer the association between the 21 case-level variables (excluding the timing of hospital admission) with a binary indicator for a 'delayed' hospital admission. Here, a delayed hospital admission was defined using a binary indicator to indicate whether each patient's hospital admission delay was greater than the population median delay.

\section{RESULTS}

A total of 14150 patients with COVID-19 were included in the CHESS database between 8 February and 13 April 2020. After applying the exclusion criteria, 6068 unique individuals were included in the final analysis (figure 1). Of these 6068 patients, 843 patients (14\%) developed symptoms of COVID-19 while a hospital inpatient, whereas 5225 patients $(86 \%)$ were admitted to hospital after exhibiting COVID-19 symptoms, with a mean interval of 4.6 days between symptom onset and hospital admission.

A comparison of the patients included in the final analysis and in the whole CHESS population is shown in table 1. In general, the analysis cohort appears representative of all those included in the CHESS database during this time period; however, there are some differences in obesity, White ethnicity and the number of recorded comorbidities.

The characteristics of the included patients, according to the timing of admission, are shown in table 2. Several differences are apparent. Patients admitted after exhibiting COVID-19 symptoms were younger, more likely to be obese and more likely to be from Black, Asian and minority ethnic (BAME) backgrounds.

Kaplan-Meier survival curves for the subcohorts of patients admitted to hospital after more than 1, 2 and 3 weeks of exhibiting COVID-19 symptoms show a higher mortality risk than the overall population (figure 2, panels A-C). After adjusting for all covariates via the multivariate Cox regression model, the fitted coefficients indicate that later hospital admission is an independent and statistically significant predictor of mortality, with a hazard ratio (HR) of 1.01 (ie, a $1 \%$ elevation in mortality risk for each additional day between symptom onset and hospital admission, $\mathrm{p}<0.005$; figure 3 ). Of particular note is the observation that being hospitalised before symptom onset (which implies that the patient developed COVID-19 while a hospital inpatient) is associated with a considerably reduced risk of mortality (HR 0.52; 95\% CIs 0.45 to 0.61 ).

The observed predictors of later hospitalisation were examined using odds ratios (ORs) based on the fitted logistic regression model, and are shown in figure 4 . There is a striking association with later presentation among healthcare workers (OR 4.87; 95\% CIs 3.31 to 6.53). Obese individuals and those who from a BAME background were also more likely to present later to hospital services.

\section{DISCUSSION}

We have shown that later hospital admission is associated with an increased risk of mortality following infection with COVID-19. Later admission is a predictor of poor outcome even after adjustment for age, sex, ethnicity, obesity and comorbidities, and suggests that timely hospital admission could reduce mortality in selected individuals who are at high risk of complications or showing signs of deterioration. We have also shown that certain characteristics

Table 2 Characteristics of the included patients, according to the timing of admission

\begin{tabular}{llll}
\hline & Hospitalised before symptom onset & Hospitalised after symptom onset & All patients \\
\hline Median age & 79 years & 64 years & 68 years \\
Male sex & $52 \%$ & $62 \%$ & $61 \%$ \\
White ethnicity & $63 \%$ & $57 \%$ & $59 \%$ \\
Asian ethnicity & $3 \%$ & $7 \%$ & $7 \%$ \\
Black ethnicity & $1 \%$ & $2 \%$ & $2 \%$ \\
Number of comorbidities & $1.0 /$ patient & $1.2 /$ patient & $1.0 /$ patient \\
\hline
\end{tabular}



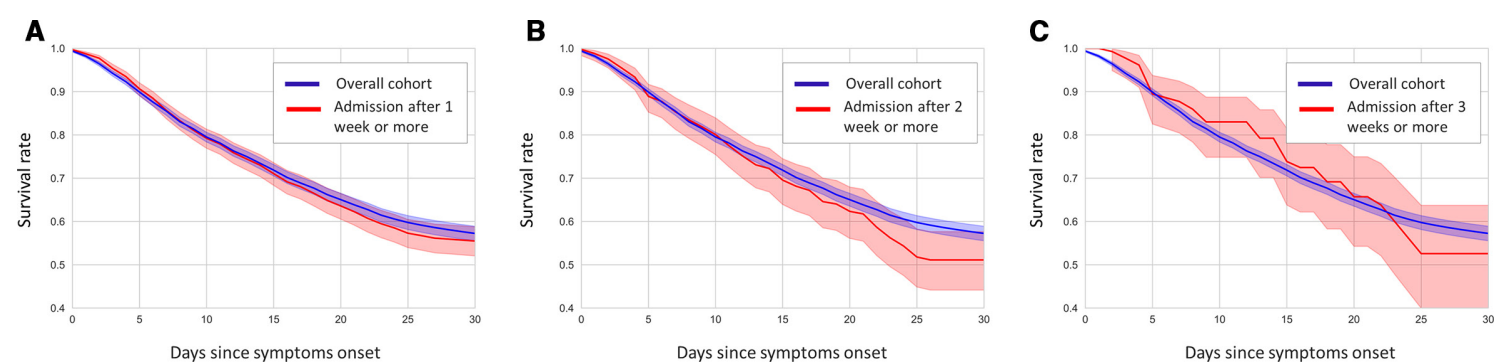

Figure 2 Kaplan-Meier survival curves for the subcohorts of patients admitted to hospital after more than 1 week (A), 2 weeks (B) and 3 weeks (C) of exhibiting COVID-19 symptoms.

are associated with later hospital admission, and identified several 'at-risk' groups to which specific interventions could be addressed.

Our findings support the hypothesis that earlier detection of deterioration and timely admission could reduce mortality, particularly as knowledge of the disease grows, and treatment modalities improve. ${ }^{67}$ It has been observed previously that early and intensive hospital intervention can improve outcomes in patients with COVID- $19 ;^{8}$ however, the mechanisms by which this occurs are not yet fully understood. Death in patients with COVID-19 is commonly caused by an unregulated immune response, sometimes described as a 'cytokine storm', ${ }^{9}$ however little is known about the cause and prevention of this phenomenon. Correction of hypoxia and physiological optimisation is likely to play a part, and recent interest has also focused on steroids and anticoagulation to reduce inflammation and the abnormal coagulation that occurs during severe COVID-19 disease. ${ }^{6}{ }^{10}$ It is therefore not surprising that hospital admission is an effective treatment for serious COVID-19 infection, and in common with other effective treatments for communicable diseases, ${ }^{11}$ the earlier it is delivered the more effective it tends to be.

The role of timely hospital admission is further supported by our observation that those patients who developed symptoms of COVID-19 while a hospital inpatient had a significantly reduced mortality. This is supported by the findings of other observational research that found patients with nosocomial infection had a lower mortality than patients who acquired COVID-19 in the community. ${ }^{12}$

We found that healthcare workers are significantly more likely to experience later hospital admission, when compared with other patients with COVID-19. This is, perhaps, intuitive, since healthcare workers may wish to avoid being a 'burden' to their colleagues, making them particularly reluctant to use hospital services. ${ }^{13}$ We also observed a strong association with later admission among individuals from a BAME background, as well as those with obesity. Later presentation by people in these groups could be a factor in the excess mortality that has been reported in previous analyses, ${ }^{14-16}$ and this is further supported by research from the USA identifying that minority ethnic patients admitted with confirmed COVID-19 infection are more likely to present with increased disease severity on admission chest radiographs. ${ }^{17}$ Strategies designed to encourage individuals from these groups to seek help at an earlier stage may prove effective in reducing these disparities.

This study has several limitations. Of 13749 patients with a confirmed diagnosis of COVID-19, $7666(56 \%)$ had no recorded date of symptom onset, and the exclusion

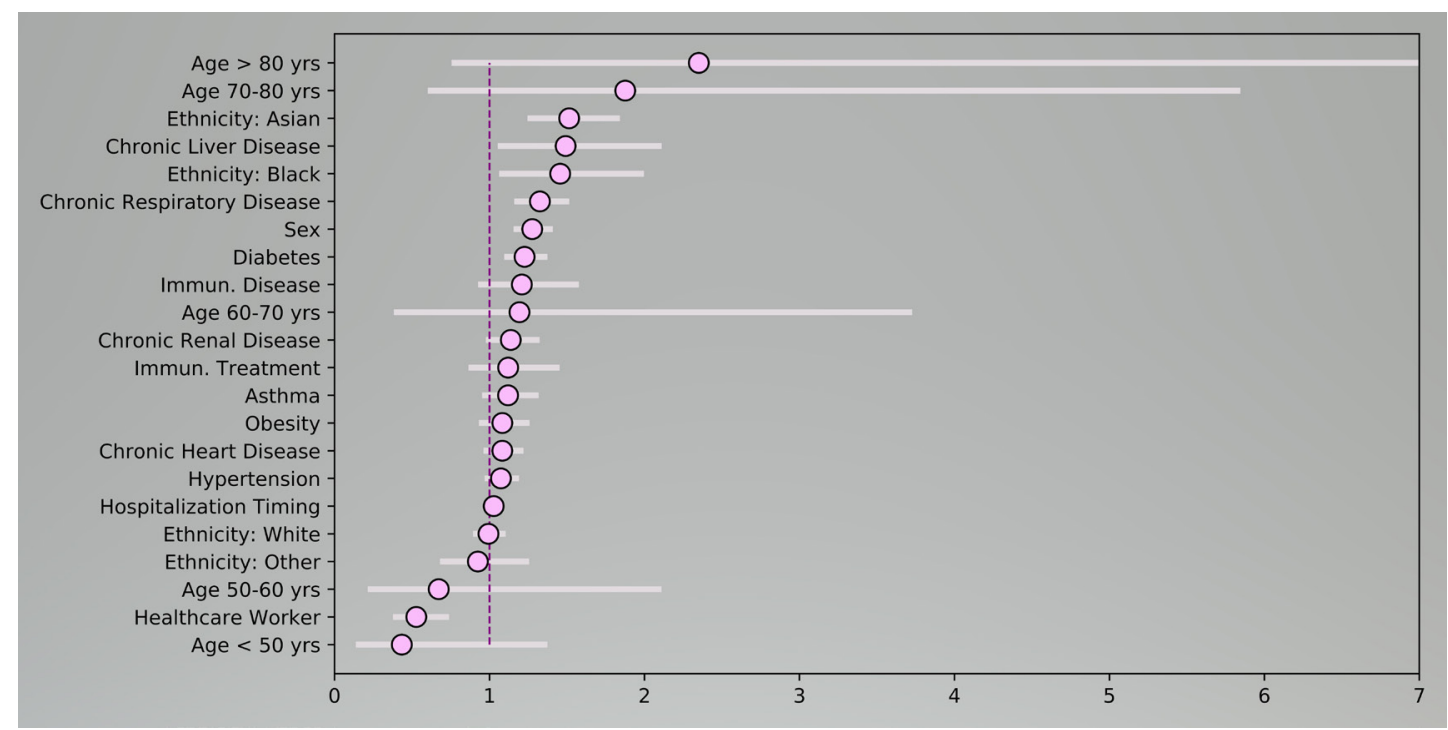

Figure 3 Hazard ratios for mortality (based on a Cox proportional hazards regression model). 


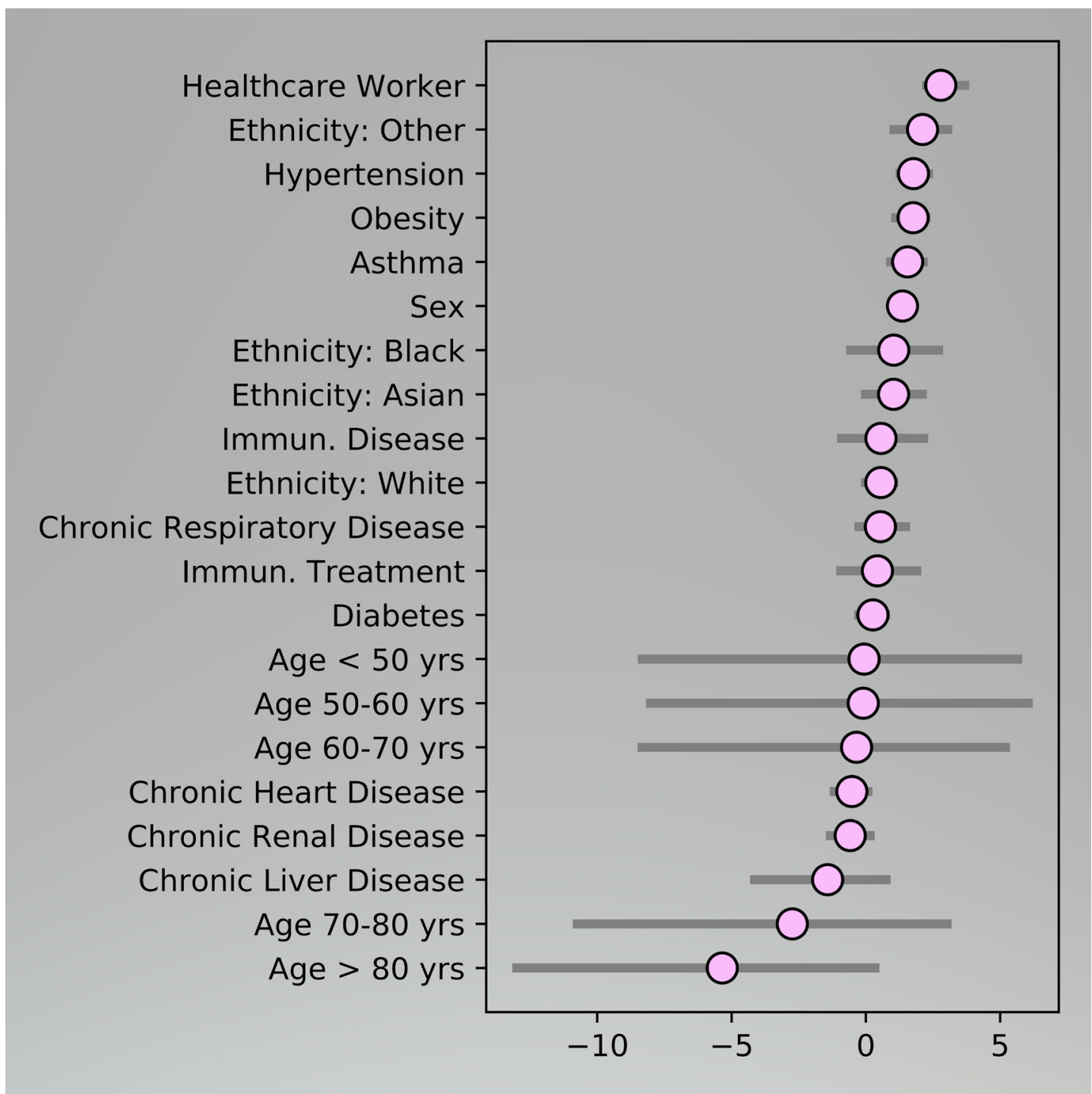

Figure 4 Predictors of later hospital admission.

of a further 15 patients with a date of symptom onset more than 3 months from hospital admission suggests weaknesses in the quality of the data recorded in CHESS. While the date of hospital admission was objectively verifiable, the date of symptom onset was self-reported and may have been subject to recall bias. We believe our findings are generalisable to adults diagnosed with COVID-19 and admitted to hospital; however, there were too few children in the sample to draw conclusions regarding the paediatric population. The most significant weakness is the use of observational data which makes it possible that our findings were influenced by unmeasured confounders.

We have demonstrated an association between later hospital admission and subsequent mortality for patients diagnosed with COVID-19. However, for the majority of individuals, COVID-19 is a mild illness, ${ }^{18}$ and it would not be practical to admit all diagnosed cases. We therefore propose further studies to evaluate the effect of identifying and monitoring high-risk patients in the community following diagnosis. It has been suggested that home measurements of oxygen saturation, using a simple pulse oximeter, could detect 'silent hypoxia' at an early stage, ${ }^{7}$ and early trials of this approach have been encouraging. ${ }^{19}$ A policy of prompt hospital admission for those showing deterioration has the potential to improve outcomes and save lives. This should be combined with specific education of the public and particularly those groups who may present later to hospital services.

\section{CONCLUSION}

We have demonstrated an independent association between the timing of hospital admission and subsequent mortality in patients diagnosed with COVID-19. Those at greatest risk of later hospital admission are healthcare workers, people with obesity and individuals from a BAME background, and this may be a factor in the poorer outcomes that have been reported in these patient groups. Strategies to identify and admit patients showing signs of deterioration in a timely way may reduce the consequent mortality from COVID-19, and should be explored further. 
Contributors JB, JR and MvdS conceived the study, and all authors contributed to the design and execution. Data were curated by JR and analysed by AA and $Z Q$, under the supervision of MvdS. The paper was drafted by JB and the figures prepared by $A A$ and $Z Q$. All authors reviewed, revised and approved the final manuscript. JB acts as a guarantor and affirms that the manuscript is an honest, accurate and transparent account of the study being reported, and that no important aspects of the study have been omitted.

Funding The authors have not declared a specific grant for this research from any funding agency in the public, commercial or not-for-profit sectors.

Competing interests None declared.

Patient and public involvement Patients and/or the public were not involved in the design, or conduct, or reporting, or dissemination plans of this research.

Patient consent for publication Not required.

Ethics approval All analysis was performed in a secure environment within NHS Digital using a fully deidentified and non-disclosive data set. Ethics approval was therefore not required.

Provenance and peer review Not commissioned; externally peer reviewed. Data availability statement No study data or additional data are available.

Open access This is an open access article distributed in accordance with the Creative Commons Attribution Non Commercial (CC BY-NC 4.0) license, which permits others to distribute, remix, adapt, build upon this work non-commercially, and license their derivative works on different terms, provided the original work is properly cited, appropriate credit is given, any changes made indicated, and the use is non-commercial. See: http://creativecommons.org/licenses/by-nc/4.0/.

ORCID iD

Jonathan Benger http://orcid.org/0000-0001-6131-0916

\section{REFERENCES}

1 World Health Organization. Novel Coronavirus (2019-nCoV) Situation Report - 1 (21st January 2020), 2020. Available: https://www.who.int/ docs/default-source/coronaviruse/situation-reports/20200121-sitrep1-2019-ncov.pdf?sfvrsn=20a99c10_ HYPERLINK [Accessed 14 Jun 2020].

2 Johns Hopkins University. COVID-19 Dashboard by the center for systems science and engineering (CSSE) at Johns Hopkins University, 2020. Available: https://coronavirus.jhu.edu/map.html [Accessed 14 Jun 2020].

3 Ottestad W, Søvik S. COVID-19 patients with respiratory failure: what can we learn from aviation medicine? Br J Anaesth 2020;125:e280-1.
4 The Telegraph. The story behind 'Stay Home, Protect the NHS, Save Lives' - the slogan that was 'too successful', 2020. Available: https:// www.telegraph.co.uk/politics/2020/05/01/story-behind-stay-homeprotect-nhs-save-lives/ [Accessed 14 Jun 2020].

5 Lynn RM, Avis JL, Lenton S, et al. Delayed access to care and late presentations in children during the COVID-19 pandemic: a snapshot survey of 4075 paediatricians in the UK and ireland. Arch Dis Child;25:archdischild-2020-319848.

6 The RECOVERY Collaborative Group. Dexamethasone in hospitalized patients with COVID-19 - preliminary report. NEJM 2020.

7 Levitan RM. Pulse oximetry as a biomarker for early identification and hospitalization of COVID-19 pneumonia. Acad Emerg Med 2020;27:785-6.

8 Sun Q, Quu H, Huang M, et al. Lower mortality of COVID-19 by early recognition and intervention: experience from Jiangsu Province. Ann Intensive Care 2020;10:33.

9 Caricchio R, Gallucci M, Dass C, et al. Preliminary predictive criteria for COVID-19 cytokine storm. Ann Rheum Dis.

10 Paranjpe I, Fuster V, Lala A, et al. Association of treatment dose anticoagulation with in-hospital survival among hospitalized patients with COVID-19. J Am Coll Cardiol 2020;76:122-4.

11 Seymour CW, Gesten F, Prescott HC, et al. Time to treatment and mortality during mandated emergency care for sepsis. N Engl J Med 2017;376:2235-44.

12 Carter B, Collins JT, Barlow-Pay F, et al. Nosocomial COVID-19 infection: examining the risk of mortality. The COPE-Nosocomial study (COVID in older people). J Hosp Infect 2020;106:376-84.

13 Davidson SK, Schattner PL. Doctors' health-seeking behaviour: a questionnaire survey. Med J Aust 2003;179:302-5.

14 Public Health England. COVID-19: review of disparities in risks and outcomes, 2020. Available: https://www.gov.uk/government/ publications/covid-19-review-of-disparities-in-risks-and-outcomes [Accessed 14 Jun 2020].

15 Sattar N, McInnes IB, McMurray JJV. Obesity a risk factor for severe COVID-19 infection: multiple potential mechanisms. Circulation 2020;06.

16 Townsend MJ, Kyle TK, Stanford FC. Outcomes of COVID-19: disparities in obesity and by ethnicity/race. Int $J$ Obes 2020;44:1807-9.

17 Joseph NP, Reid NJ, Som A, et al. Racial/Ethnic disparities in disease severity on admission chest radiographs among patients admitted with confirmed COVID-19: a retrospective cohort study. Radiology 2020:202602.

18 Tabata S, Imai K, Kawano S, et al. Clinical characteristics of COVID-19 in 104 people with SARS-CoV-2 infection on the diamond Princess cruise SHIP: a retrospective analysis. Lancet Infect Dis 2020;20:1043-50.

19 Annis T, Pleasants S, Hultman G, et al. Rapid implementation of a COVID-19 remote patient monitoring program. J Am Med Inform Assoc 2020;27:1326-30. 